\title{
Kocuria atrinae sp. nov., isolated from traditional Korean fermented seafood
}

\author{
Eun-Jin Park, Min-Soo Kim, Seong Woon Roh, Mi-Ja Jung \\ and Jin-Woo Bae
}

Correspondence

Jin-Woo Bae

baejw@khu.ac.kr

\author{
Department of Life and Nanopharmaceutical Sciences and Department of Biology, Kyung Hee \\ University, Seoul 130-701, Republic of Korea
}

\begin{abstract}
A novel actinobacterium, strain $\mathrm{P}^{3} 0^{\top}$, was isolated from jeotgal, a traditional Korean fermented seafood. Cells were aerobic, Gram-positive, non-motile and coccoid. Optimal growth occurred at $30-37{ }^{\circ} \mathrm{C}$, at $\mathrm{pH} 8-9$ and in the presence of $0-2 \%(\mathrm{w} / \mathrm{v}) \mathrm{NaCl}$. Based on 16S rRNA gene sequence analysis, strain $\mathrm{P} 30^{\top}$ was phylogenetically closely related to Kocuria carniphila, Kocuria gwangalliensis, Kocuria rhizophila, Kocuria marina, Kocuria rosea and K. varians with levels of similarity of $98.6,98.2,98.1,97.4,97.3$ and $97.3 \%$, respectively, to the type strains of these species. Levels of DNA-DNA relatedness between strain $\mathrm{P}_{30}{ }^{\top}$ and the type strains of $K$. carniphila, K. rhizophila, K. marina, K. rosea and $K$. varians were 37, 43, 37, 25 and 17\%, respectively. The predominant menaquinone of strain $\mathrm{P}_{30}{ }^{\top}$ was $\mathrm{MK}-7$. Major cellular fatty acids were anteiso- $C_{15: 0}$, iso- $C_{15: 0}$ and iso- $C_{16: 0}$. The genomic DNA G $+C$ content of strain $\mathrm{P}_{30}{ }^{\top}$ was $70.2 \mathrm{~mol} \%$. Based on these data, strain $\mathrm{P}_{30}{ }^{\top}$ is considered to represent a novel species of the genus Kocuria, for which the name Kocuria atrinae sp. nov. is proposed. The type strain is $\mathrm{P} 30^{\top}\left(=\right.$ KCTC $19594^{\top}=$ JCM $\left.15914^{\mathrm{T}}\right)$.
\end{abstract}

The genus Kocuria within the family Actinobacteria was first described by Stackebrandt et al. (1995), and at the time of writing comprises 16 recognized species, namely Kocuria rosea, K. varians, K. kristinae (Stackebrandt et al., 1995), K. erythromyxa (Rainey et al., 1997), K. palustris, K. rhizophila (Kovacs et al., 1999), K. polaris (Reddy et al., 2003), K. marina (Kim et al., 2004), K. carniphila (Tvrzová et al., 2005), K. aegyptia (Li et al., 2006), K. himachalensis (Mayilraj et al., 2006), K. flava, K. turfanensis (Zhou et al., 2008), K. gwangalliensis (Seo et al., 2009), K. halotolerans (Tang et al., 2009) and K. koreensis (Park et al., 2010). Strains of these species have been isolated from desert soil, saline water, seawater, air, marine sediment, the rhizoplane of narrowleaved cattail, a cold desert soil of the Indian Himalayas and fermented seafood (Stackebrandt et al., 1995; Rainey et al., 1997; Kovacs et al., 1999; Reddy et al., 2003; Kim et al., 2004; Tvrzová et al., 2005; Mayilraj et al., 2006; Li et al., 2006; Zhou et al., 2008; Seo et al., 2009; Tang et al., 2009; Park et al., 2010). In the present study, we describe the detailed phylogenetic characterization of a novel species of the genus Kocuria.

Strain $\mathrm{P} 30^{\mathrm{T}}$ was isolated from a Korean traditional fermented seafood by using the standard dilution-plating method on marine agar 2216 (MA; BBL) medium at $30{ }^{\circ} \mathrm{C}$ and was transferred several times to obtain a pure culture.

The GenBank/EMBL/DDBJ accession number for the 16S rRNA gene sequence of strain $\mathrm{P} 30^{\top}$ is FJ607311.
Salinity tolerance and requirement were investigated in identical marine broth (MB; BBL) medium without $\mathrm{NaCl}$ and with $\mathrm{NaCl}$ at various concentrations $(1,2,3$ and 5$30 \%, w / v$, at intervals of $5 \%$ ). For determination of the optimum culture conditions for strain $\mathrm{P} 30^{\mathrm{T}}$, growth was tested at $4,10,15,20,25,30,37$ and $40{ }^{\circ} \mathrm{C}$ on MA medium and at $\mathrm{pH} 3-10$ (at intervals of $1.0 \mathrm{pH}$ unit) in $\mathrm{MB}$ medium adjusted by the addition of $\mathrm{HCl}$ or $\mathrm{NaOH}$ (prior to sterilization). Morphological and physiological studies for strain $\mathrm{P} 30^{\mathrm{T}}$ were performed with cells grown on MA medium at $30{ }^{\circ} \mathrm{C}$ for 2 days. Growth under anaerobic conditions was tested after 7 days incubation at $30{ }^{\circ} \mathrm{C}$ on MA medium in an oxygen-free $\mathrm{N}_{2} / \mathrm{CO}_{2} / \mathrm{H}_{2} \quad(8: 1: 1)$ anaerobic chamber. Gram staining reaction was performed by using a Gram Stain kit (BBL). Cell morphology of strain $\mathrm{P} 30^{\mathrm{T}}$ was observed by light microscopy (E600; Nikon). Semisolid agar was used for tests of motility (Tittsler \& Sandholzer, 1936). Oxidase and catalase activities were determined by using a $3 \%(\mathrm{v} / \mathrm{v}) \mathrm{H}_{2} \mathrm{O}_{2}$ solution and an oxidase reagent (bioMérieux), respectively. Activity of 19 hydrolytic enzymes was determined by using an API ZYM kit (bioMérieux). Substrate utilization by strain $\mathrm{P} 30^{\mathrm{T}}$ was tested by using API 20NE (bioMérieux) and Biolog GP2 metabolic fingerprinting plates (Biolog).

Colonies grown on MA medium were pale yellow, circular, smooth and opaque. Cells of strain $\mathrm{P} 30^{\mathrm{T}}$ were aerobic, Gram-positive, non-motile and coccoid $(1.0-1.5 \mu \mathrm{m}$ in diameter). The strain was able to grow at $25-37^{\circ} \mathrm{C}$, at 
pH 7-9 and in the presence of $0-4 \% \mathrm{NaCl}$. Strain $\mathrm{P} 30^{\mathrm{T}}$ and all reference strains tested were catalase-positive and oxidase-negative (Stackebrandt et al., 1995; Kovacs et al., 1999; Kim et al., 2004; Tvrzová et al., 2005). The morphological, cultural, physiological and biochemical characteristics of strain $\mathrm{P} 30^{\mathrm{T}}$ and the type strains of five closely related Kocuria species are summarized in Table 1.

Chromosomal DNA was extracted and purified according to the method described by Sambrook et al. (1989). The $16 \mathrm{~S}$ rRNA gene was amplified and purified by using a PCR Pre-Mix (Solgent) and purification kit (Solgent), respectively. $16 \mathrm{~S}$ rRNA gene sequencing and phylogenetic analysis were performed as described by Roh et al. (2008). Phylogenetic relationships between strain $\mathrm{P} 30^{\mathrm{T}}$ and representatives of the genus Kocuria were performed by using MEGA 4 (Tamura et al., 2007). Levels of $16 \mathrm{~S}$ rRNA gene sequence similarity were determined by using the EzTaxon server (www.eztaxon.org; Chun et al., 2007). Distance matrices were computed based on the method described by Kimura (1980) and were used to construct dendrograms by the neighbour-joining (Saitou \& Nei, 1987) and maximum-parsimony (Kluge \& Farris, 1969) methods. Bootstrap analysis was conducted with 1000 replicates to evaluate the stability of the trees. DNA-DNA hybridization experiments were performed by using photobiotin-labelled DNA probes and microwell plates as described by Ezaki et al. (1989).

As shown in the phylogenetic tree based on the neighbourjoining method (Fig. 1), strain $\mathrm{P} 30^{\mathrm{T}}$ formed a monophyletic cluster with K. carniphila CCM $132^{\mathrm{T}}$. On the basis of $16 \mathrm{~S}$ rRNA gene sequences, strain $\mathrm{P} 30^{\mathrm{T}}$ was a member of the genus Kocuria and was related most closely to K. carniphila CCM $132^{\mathrm{T}}$, K. gwangalliensis DE $706^{\mathrm{T}}$, K. rhizophila DSM $11926^{\mathrm{T}}$, K. marina KMM $3905^{\mathrm{T}}, K$. rosea DSM $20447^{\mathrm{T}}$ and K. varians DSM $20033^{\mathrm{T}}$ at levels of similarity of $98.4,98.2$, 98.1, 97.5, 97.3 and 97.3\%, respectively. Levels of DNADNA relatedness between strain $\mathrm{P} 30^{\mathrm{T}}$ and its five closest relatives (16S rRNA gene sequence similarity $>97.0 \%$ ), namely K. carniphila CCM $132^{\mathrm{T}}$, K. rhizophila DSM $11926^{\mathrm{T}}$, K. marina KMM $3905^{\mathrm{T}}$, K. rosea DSM $20447^{\mathrm{T}}$ and K. varians DSM $20033^{\mathrm{T}}$, were $37,43,37,25$ and $17 \%$, respectively. These values are clearly below the $70 \%$ recommended cutoff for the delineation of bacterial species (Stackebrandt \& Goebel, 1994; Wayne et al., 1987). The low levels of DNADNA relatedness clearly confirmed that strain $\mathrm{P} 30^{\mathrm{T}}$ represents a novel species.

The genomic DNA G $+\mathrm{C}$ content of strain $\mathrm{P} 30^{\mathrm{T}}$ was determined according to the fluorimetric method by using SYBR Green I as described by Gonzalez \& Saiz-Jimenez (2002). Cell biomass for analyses of cellular fatty acids and menaquinones was obtained from cultures grown on MA for 2 days at $30{ }^{\circ} \mathrm{C}$. For quantitative analysis of cellular fatty acids, cells of $\mathrm{P} 30^{\mathrm{T}}$ and of K. carniphila CCM $132^{\mathrm{T}}, K$. rhizophila DSM $11926^{\mathrm{T}}$ and K. varians DSM $20033^{\mathrm{T}}$ were tested. Fatty acids were saponified, methylated and extracted by using the standard protocol provided by the MIDI/Hewlett Packard Microbial Identification System
(Sasser, 1990), and were analysed by GC (Hewlett Packard 6890). TLC was used for analysis of menaquinones, as described by Hiraishi et al. (1996). Three replicate trials for each experiment were performed.

The $\mathrm{G}+\mathrm{C}$ content of the genomic DNA of strain $\mathrm{P} 30^{\mathrm{T}}$ was $70.2 \mathrm{~mol} \%$, within the range of $66-75 \mathrm{~mol} \%$ reported for

Table 1. Differential characteristics between strain $P 30^{\top}$ and closely related species of the genus Kocuria

Strains: $1, \mathrm{P} 30^{\mathrm{T}} ; 2$, K. carniphila CCM $132^{\mathrm{T}} ; 3$, K. rhizophila DSM $11926^{\mathrm{T}}$; 4, K. varians DSM $20033^{\mathrm{T}}$; 5, K. marina KMM $3905^{\mathrm{T}}$; 6, K. rosea DSM $20447^{\mathrm{T}}$. Data for $1-3$ are from the present study; data for 4-6 are from Stackebrandt et al. (1995), Kim et al. (2004) and Tvrzová et al. (2005). +, Positive; -, negative; w, weakly positive; NR, not reported.

\begin{tabular}{|c|c|c|c|c|c|c|}
\hline Characteristic & 1 & 2 & 3 & 4 & 5 & 6 \\
\hline \multicolumn{7}{|l|}{ Growth at/with: } \\
\hline $5{ }^{\circ} \mathrm{C}$ & - & - & - & - & + & - \\
\hline $10 \% \mathrm{NaCl}$ & + & - & + & - & + & - \\
\hline $15 \% \mathrm{NaCl}$ & - & - & - & - & + & - \\
\hline Reduction of nitrates to nitrites & + & + & - & - & + & + \\
\hline L-Arginine dihydrolase & + & - & - & - & - & - \\
\hline Urease & + & - & - & + & + & - \\
\hline Aesculin hydrolysis & + & - & - & - & NR & $\mathrm{w}$ \\
\hline Gelatin hydrolysis & - & - & + & + & + & - \\
\hline Starch hydrolysis & + & - & - & - & - & + \\
\hline Alkaline phosphatase & - & + & + & - & - & - \\
\hline$\beta$-Galactosidase & - & + & - & + & + & - \\
\hline$\beta$-Glucuronidase & - & - & - & - & + & - \\
\hline \multicolumn{7}{|l|}{ Utilization of: } \\
\hline Dextrin & + & + & + & + & NR & - \\
\hline Glycogen & - & - & + & + & NR & - \\
\hline Tween 40 & + & + & + & + & + & - \\
\hline Tween 80 & + & - & + & + & - & - \\
\hline$N$-Acetyl-D-glucosamine & - & - & + & - & - & - \\
\hline$N$-Acetyl- $\beta$-D-mannosamine & - & + & - & - & - & - \\
\hline L-Arabinose & - & + & + & + & - & + \\
\hline L-Fucose & - & + & + & + & + & + \\
\hline D-Galacturonic acid & - & - & - & - & NR & - \\
\hline myo-Inositol & - & + & - & - & - & - \\
\hline Maltose & + & - & - & NR & + & - \\
\hline D-Mannitol & - & + & - & - & - & + \\
\hline Melibiose & - & + & - & + & - & - \\
\hline Methyl- $\alpha$-D-galactoside & - & + & - & - & NR & - \\
\hline 3-Methylglucose & - & - & - & $\mathrm{w}$ & NR & - \\
\hline D-Sorbitol & + & + & - & + & - & + \\
\hline Xylitol & + & - & + & + & NR & - \\
\hline$\beta$-Hydroxybutyric acid & + & + & - & + & NR & - \\
\hline D-Malic acid & - & - & + & + & NR & + \\
\hline $\mathrm{N}$-Acetyl-L-glutamic acid & + & - & - & + & NR & - \\
\hline L-Glutamic acid & + & + & - & - & NR & - \\
\hline Glycerol & + & + & - & - & NR & + \\
\hline Adenosine & + & + & - & + & NR & - \\
\hline Uridine & + & - & + & + & NR & $\mathrm{w}$ \\
\hline D-Glucose 6-phosphate & - & - & - & + & NR & $\mathrm{w}$ \\
\hline
\end{tabular}




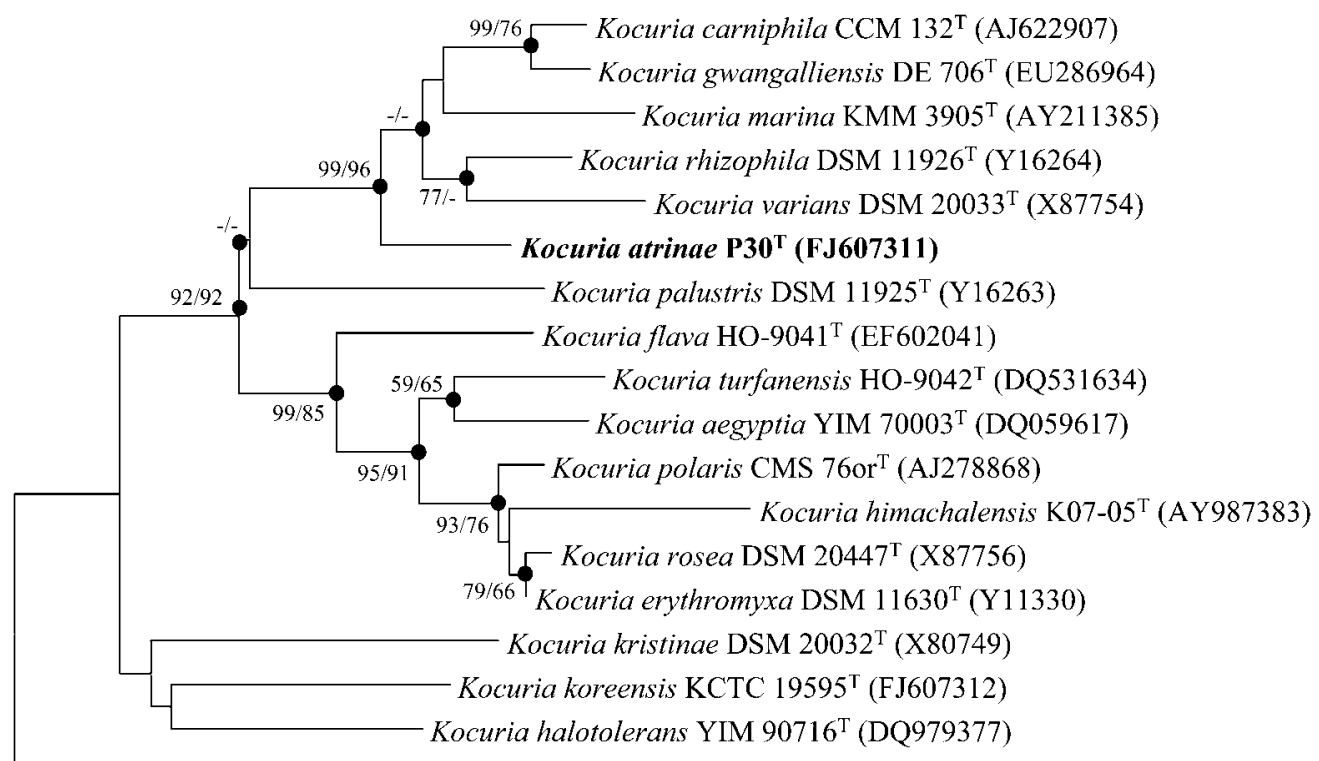

Nesterenkonia aethiopica DSM $17733^{\mathrm{T}}$ (AY574575)

0.005

Fig. 1. Neighbour-joining phylogenetic consensus tree based on $16 \mathrm{~S}$ rRNA gene sequences showing the relationship between strain $\mathrm{P} 30^{\top}$ and representative members of the genus Kocuria. Filled circles indicate generic branches that were also recovered with the maximum-parsimony method. Nesterenkonia aethiopica DSM $17733^{\top}$ served as an outgroup. Numbers at nodes are bootstrap values calculated from neighbour-joining/maximum-parsimony probabilities as percentages of 1000 replications; only values $>50 \%$ are shown. GenBank accession numbers are shown in parentheses. Bar, 0.005 substitutions per nucleotide position.

members of the genus Kocuria (Stackebrandt et al., 1995; Rainey et al., 1997; Kovacs et al., 1999; Reddy et al., 2003; Kim et al., 2004; Tvrzová et al., 2005; Mayilraj et al., 2006; Li et al., 2006; Zhou et al., 2008). The cellular fatty acid profiles of strain $\mathrm{P} 30^{\mathrm{T}}$ and the type strains of five related Kocuria species are shown in Table 2. Strain $\mathrm{P} 30^{\mathrm{T}}$ contained the following cellular fatty acids $(>1.0 \%$ of the total): anteiso- $\mathrm{C}_{15: 0}(53.7 \%)$, iso- $\mathrm{C}_{15: 0}(14.1 \%)$, iso$\mathrm{C}_{16: 0}(10.3 \%)$, anteiso- $\mathrm{C}_{17: 0}(7.2 \%), \mathrm{C}_{16: 0}(4.8 \%)$, iso$\mathrm{C}_{14: 0}(4.4 \%)$ and $\mathrm{C}_{14: 0}(1.5 \%)$. A large proportion of anteiso- $\mathrm{C}_{15: 0}$ was also reported for some Kocuria species (Stackebrandt et al., 1995; Rainey et al., 1997; Kovacs et al., 1999; Reddy et al., 2003; Kim et al., 2004; Tvrzová et al., 2005; Mayilraj et al., 2006; Li et al., 2006; Zhou et al., 2008). The predominant menaquinone of strain $\mathrm{P} 30^{\mathrm{T}}$ was MK-7.

Taken together, these phenotypic, genotypic and phylogenetic characteristics indicate that strain $\mathrm{P} 30^{\mathrm{T}}$ represents a novel species of the genus Kocuria, for which the name Kocuria atrinae sp. nov. is proposed.

\section{Description of Kocuria atrinae sp. nov.}

Kocuria atrinae (a.tri'na.e. N.L. n. Atrina a scientific zoological genus; N.L. gen. n. atrinae of Atrina, isolated from Atrina pectinata).

Cells are Gram-positive, aerobic, non-motile and coccoid with a diameter of $1.0-1.5 \mu \mathrm{m}$. Colonies are pale yellow, circular, smooth and opaque after 2 days incubation on MA at $30{ }^{\circ} \mathrm{C}$. Cells grow at $25-37{ }^{\circ} \mathrm{C}$, at pH 7-9 and in the presence of $0-4 \% \mathrm{NaCl}$. Optimal growth conditions on MA or in MB medium are $30-37^{\circ} \mathrm{C}, \mathrm{pH} 8-9$ and $0-2 \%$ $\mathrm{NaCl}$. Catalase-positive and oxidase-negative. Reduces nitrate to nitrite, does not produce indole and is unable to ferment D-glucose. Positive for L-arginine dihydrolase, urease, esterase lipase (C8), leucine arylamidase, naphtholAS-BI-phosphohydrolase and $\alpha$-glucosidase. Negative for alkaline phosphatase, esterase (C4), valine arylamidase, cystine arylamidase, trypsin, $\alpha$-chymotrypsin, acid phosphatase, $\alpha$-galactosidase, $\beta$-galactosidase, $\beta$-glucuronidase, $\beta$-glucosidase, $\alpha$-mannosidase and $\alpha$-fucosidase. Hydrolyses aesculin but not gelatin or PNPG. According to growth on Biolog GP2 plates, assimilates dextrin, Tweens 40 and 80 , D-fructose, D-gluconic acid, $\alpha$-D-glucose, maltose, maltotriose, D-mannose, D-psicose, D-ribose, D-sorbitol, trehalose, turanose, xylitol, acetic acid, $\alpha$-hydroxybutyric acid, $\beta$-hydroxybutyric acid, $p$-hydroxyphenylacetic acid, $\alpha$-ketoglutaric acid, $\alpha$-ketovaleric acid, D-lactic acid methyl ester, L-malic acid, pyruvic acid methyl ester, succinic acid monomethyl ester, propionic acid, pyruvic acid, succinic acid, $\mathrm{N}$-acetyl-L-glutamic acid, L-asparagine, L-glutamic acid, putrescine, glycerol, adenosine, 2 -deoxyadenosine, inosine, thymidine, uridine, thymidine $5^{\prime}$-monophosphate and DL- $\alpha$-glycerol phosphate, but not $\alpha$-cyclodextrin, $\beta$-cyclodextrin, glycogen, inulin, mannan, $N$-acetyl-D-glucosamine, $N$-acetyl- $\beta$-D-mannosa- 
Table 2. Cellular fatty acid profiles of strain $\mathrm{P} 30^{\top}$ and closely related species of the genus Kocuria

Strains: $1, \mathrm{P} 30^{\mathrm{T}}$ (grown on MA); 2, K. carniphila CCM $132^{\mathrm{T}}$ (MA); 3, K. rhizophila DSM $11926^{\mathrm{T}}$ (MA); 4, K. varians DSM $20033^{\mathrm{T}}$ (MA); 5, K. marina KMM $3905^{\mathrm{T}}$ (MA); 6, K. rosea DSM $20447^{\mathrm{T}}$ (trypticase soy broth). Data for 1-4 are from the present study; data for 5 and 6 are from Kim et al. (2004) and Stackebrandt et al. (1995), respectively. Values are percentages of the total fatty acids. $\operatorname{tr}$, Trace $(<1.0 \%) ;-$, not detected.

\begin{tabular}{|c|c|c|c|c|c|c|}
\hline Fatty acid & 1 & 2 & 3 & 4 & 5 & 6 \\
\hline $\mathrm{C}_{12: 0}$ & $\operatorname{tr}$ & $\operatorname{tr}$ & - & - & - & - \\
\hline iso- $\mathrm{C}_{13: 0}$ & $\operatorname{tr}$ & - & $\operatorname{tr}$ & $\operatorname{tr}$ & - & - \\
\hline anteiso- $\mathrm{C}_{13: 0}$ & $\operatorname{tr}$ & $\operatorname{tr}$ & $\operatorname{tr}$ & $\operatorname{tr}$ & - & - \\
\hline iso- $\mathrm{C}_{14: 0}$ & 4.4 & 2.4 & 1.9 & $\operatorname{tr}$ & 1.9 & 1.8 \\
\hline $\mathrm{C}_{14: 0}$ & 1.5 & 1.8 & 1.3 & 1.7 & 2.3 & 1.5 \\
\hline iso- $\mathrm{C}_{15: 0}$ & 14.1 & 3.1 & 12.4 & 4.3 & 2.5 & 7.6 \\
\hline anteiso- $\mathrm{C}_{15: 0}$ & 53.7 & 65.3 & 47.7 & 54.2 & 74.0 & 70.9 \\
\hline $\mathrm{C}_{15: 0}$ & $\operatorname{tr}$ & $\operatorname{tr}$ & - & 1.0 & - & - \\
\hline $\mathrm{C}_{16: 1}$ & - & - & - & - & - & 5.9 \\
\hline iso- $\mathrm{C}_{16: 0}$ & 10.3 & 4.3 & 9.7 & 6.9 & 7.0 & 1.6 \\
\hline$C_{16: 0}$ & 4.8 & 7.0 & 2.3 & 11.6 & 2.9 & 1.6 \\
\hline anteiso- $\mathrm{C}_{17: 1}$ & - & - & - & - & 3.0 & - \\
\hline iso- $\mathrm{C}_{17: 0}$ & $\operatorname{tr}$ & $\operatorname{tr}$ & 1.6 & - & - & 1.3 \\
\hline anteiso- $\mathrm{C}_{17: 0}$ & 7.2 & 10.7 & 20.8 & 8.4 & 4.3 & 16.0 \\
\hline $\mathrm{C}_{18: 1} \omega 9 c$ & - & $\operatorname{tr}$ & - & - & - & $\operatorname{tr}$ \\
\hline $\mathrm{C}_{18: 0}$ & $\operatorname{tr}$ & 1.8 & - & - & - & $\operatorname{tr}$ \\
\hline
\end{tabular}

mine, amygdalin, L-arabinose, D-arabitol, arbutin, cellobiose, L-fucose, D-galactose, D-galacturonic acid, gentiobiose, myo-inositol, $\alpha$-D-lactose, lactulose, D-mannitol, melezitose, melibiose, methyl- $\alpha$-D-galactoside, methyl- $\beta$ D-galactoside, 3-methylglucose, methyl- $\alpha$-D-glucoside, methyl- $\beta$-D-glucoside, methyl- $\alpha$-D-mannoside, palatinose, raffinose, L-rhamnose, sedoheptulosan, stachyose, sucrose, D-tagatose, D-xylose, $\gamma$-hydroxybutyric acid, lactamide, Dmalic acid, L-malic acid, succinamic acid, L-alaninamide, D-alanine, L-alanine, L-alanylglycine, glycyl-L-glutamic acid, L-pyroglutamic acid, L-serine, 2,3-butanediol, adenosine $5^{\prime}$-monophosphate, uridine $5^{\prime}$-monophosphate, D-fructose 6-phosphate, $\alpha$-D-glucose 1-phosphate or D-glucose 6phosphate. The predominant menaquinone is MK-7. Major cellular fatty acids are anteiso- $\mathrm{C}_{15: 0}$, iso- $\mathrm{C}_{15: 0}$ and iso- $\mathrm{C}_{16: 0}$. The DNA $\mathrm{G}+\mathrm{C}$ content of the type strain is $70.2 \mathrm{~mol} \%$.

The type strain, $\mathrm{P} 30^{\mathrm{T}}\left(=\right.$ KCTC $\left.19594^{\mathrm{T}}=\mathrm{JCM} 15914^{\mathrm{T}}\right)$, was isolated from a traditional fermented seafood in Korea.

\section{Acknowledgements}

We thank Dr J. P. Euzéby (École Nationale Vétérinaire, France) for etymological advice. This work was supported by the Environmental Biotechnology National Core Research Center (KOSEF: R15-2003012-02002-0) and TDPAF (Technology Development Program for Agriculture and Forestry) of the Ministry for Agriculture, Forestry and Fisheries of the Republic of Korea.

\section{References}

Chun, J., Lee, J. H., Jung, Y., Kim, M., Kim, S., Kim, B. K. \& Lim, Y. W. (2007). EzTaxon: a web-based tool for the identification of prokaryotes based on 16S ribosomal RNA gene sequences. Int J Syst Evol Microbiol 57, 2259-2261.

Ezaki, T., Hashimoto, Y. \& Yabuuchi, E. (1989). Fluorometric deoxyribonucleic acid-deoxyribonucleic acid hybridization in microdilution wells as an alternative to membrane filter hybridization in which radioisotopes are used to determine genetic relatedness among bacterial strains. Int J Syst Bacteriol 39, 224-229.

Gonzalez, J. M. \& Saiz-Jimenez, C. (2002). A fluorimetric method for the estimation of $\mathrm{G}+\mathrm{C}$ mol\% content in microorganisms by thermal denaturation temperature. Environ Microbiol 4, 770-773.

Hiraishi, A., Ueda, Y., Ishihara, J. \& Mori, T. (1996). Comparative lipoquinone analysis of influent sewage and activated sludge by highperformance liquid chromatography and photodiode array detection. J Gen Appl Microbiol 42, 457-469.

Kim, S. B., Nedashkovskaya, O. I., Mikhailov, V. V., Han, S. K., Kim, K. O., Rhee, M. S. \& Bae, K. S. (2004). Kocuria marina sp. nov., a novel actinobacterium isolated from marine sediment. Int J Syst Evol Microbiol 54, 1617-1620.

Kimura, M. (1980). A simple method for estimating evolutionary rates of base substitutions through comparative studies of nucleotide sequences. J Mol Evol 16, 111-120.

Kluge, A. G. \& Farris, J. S. (1969). Quantitative phyletics and the evolution of anurans. Syst Zool 18, 1-32.

Kovacs, G., Burghardt, J., Pradella, S., Schumann, P., Stackebrandt, E. \& Màrialigeti, K. (1999). Kocuria palustris sp. nov. and Kocuria rhizophila sp. nov., isolated from the rhizoplane of the narrow-leaved cattail (Typha angustifolia). Int J Syst Bacteriol 49, 167-173.

Li, W.-J., Zhang, Y.-Q., Schumann, P., Chen, H.-H., Hozzein, W. N., Tian, X.-P., Xu, L.-H. \& Jiang, C.-L. (2006). Kocuria aegyptia sp. nov., a novel actinobacterium isolated from a saline, alkaline desert soil in Egypt. Int J Syst Evol Microbiol 56, 733-737.

Mayilraj, S., Kroppenstedt, R. M., Suresh, K. \& Saini, H. S. (2006). Kocuria himachalensis sp. nov., an actinobacterium isolated from the Indian Himalayas. Int J Syst Evol Microbiol 56, 1971-1975.

Park, E. J., Roh, S. W., Kim, M. S., Jung, M. J., Shin, K. S. \& Bae, J. W. (2010). Kocuria koreensis sp. nov. isolated from fermented seafood. Int J Syst Evol Microbiol 60, 140-143.

Rainey, F. A., Nobre, M. F., Schumann, P., Stackebrandt, E. \& Da Costa, M. S. (1997). Phylogenetic diversity of the deinococci as determined by $16 \mathrm{~S}$ ribosomal DNA sequence comparison. Int J Syst Bacteriol 47, 510-514.

Reddy, G. S. N., Prakash, J. S. S., Prabahar, V., Matsumoto, G. I., Stackebrandt, E. \& Shivaji, S. (2003). Kocuria polaris sp. nov., an orange-pigmented psychrophilic bacterium isolated from an Antarctic cyanobacterial mat sample. Int J Syst Evol Microbiol 53, 183-187.

Roh, S. W., Sung, Y., Nam, Y. D., Chang, H. W., Kim, K. H., Yoon, J. H., Jeon, C. O., Oh, H. M. \& Bae, J. W. (2008). Arthrobacter soli sp. nov., a novel bacterium isolated from wastewater reservoir sediment. J Microbiol 46, 40-44.

Saitou, N. \& Nei, M. (1987). The neighbor-joining method: a new method for reconstructing phylogenetic trees. Mol Biol Evol 4, 406425.

Sambrook, J., Fritsch, E. F. \& Maniatis, T. (1989). Molecular Cloning: a Laboratory Manual, 2nd edn. Cold Spring Harbor, NY: Cold Spring Harbor Laboratory.

Sasser, M. (1990). Identification of bacteria by gas chromatography of cellular fatty acids, MIDI Technical Note 101. Newark, DE: MIDI Inc. 
Seo, Y. B., Kim, D. E., Kim, G. D., Kim, H. W., Nam, S. W., Kim, Y. T. \& Lee, J. H. (2009). Kocuria gwangalliensis sp. nov., a novel actinobacterium isolated from seawater. Int J Syst Evol Microbiol 59, 2769-2772.

Stackebrandt, E. \& Goebel, B. M. (1994). Taxonomic note: a place for DNA-DNA reassociation and $16 \mathrm{~S}$ rRNA sequence analysis in the present species definition in bacteriology. Int J Syst Bacteriol 44, 846-849.

Stackebrandt, E., Koch, C., Gvozdiak, O. \& Schumann, P. (1995). Taxonomic dissection of the genus Micrococcus: Kocuria gen. nov., Nesterenkonia gen. nov., Kytococcus gen. nov., Dermacoccus gen. nov., and Micrococcus Cohn 1872 gen. emend. Int J Syst Bacteriol 45, 682692.

Tamura, K., Dudley, J., Nei, M. \& Kumar, S. (2007). MEGA 4: molecular evolutionary genetics analysis (MEGA) software version 4.0. Mol Biol Evol 24, 1596-1599.

Tang, S. K., Wang, Y., Lou, K., Mao, P. H., Xu, L. H., Jiang, C. L., Kim, C. J. \& Li, W. J. (2009). Kocuria halotolerans sp. nov., a novel actinobacterium isolated from a saline soil in China. Int J Syst Evol Microbiol 59, 1316-1320.

Tittsler, R. P. \& Sandholzer, L. A. (1936). Use of semi-solid agar for the detection of bacterial motility. J Bacteriol 31, 575-580.

Tvrzová, L., Schumann, P., Sedlácek, I., Pácová, Z., Spröer, C., Verbarg, S. \& Kroppenstedt, R. M. (2005). Reclassification of strain CCM 132, previously classified as Kocuria varians, as Kocuria carniphila sp. nov. Int J Syst Evol Microbiol 55, 139-142.

Wayne, L. G., Brenner, D. J., Colwell, R. R., Grimont, P. A. D., Kandler, O., Krichevsky, M. I., Moore, L. H., Moore, W. E. C., Murray, R. G. E. \& other authors (1987). International Committee on Systematic Bacteriology. Report of the ad hoc committee on reconciliation of approaches to bacterial systematics. Int J Syst Bacteriol 37, 463-464.

Zhou, G., Luo, X., Tang, Y., Zhang, L., Yang, Q., Qio, Y. \& Fang, C. (2008). Kocuria flava sp. nov. and Kocuria turfanensis sp. nov., airborne actinobacteria isolated from Xinjiang, China. Int J Syst Evol Microbiol 58, 1304-1307. 